\title{
BRAIDS FOR PRETZEL KNOTS
}

\author{
M. SUFFCZYŃSKI \\ Institute of Physics, Polish Academy of Sciences \\ Al. Lotników 32/46, 02-668 Warsaw, Poland \\ (Received October 26, 2000)
}

\begin{abstract}
The words of braid closures for the pretzel knots, in particular the twist knots relevant to molecular supercoils in biochemistry, are recorded in a standardized form, which enables one to see the regular pattern of the words, and thus to write the braid words representing pretzel knots for general values of the crossing number.
\end{abstract}

PACS numbers: 02.40.-k, 36.20.-r, 87.10.+e, 87.15.-v

\section{Introduction}

The braid closures are useful for representing knots [1-4]. In the published tabulation of Jones [2], of braid words for braid closures representing all knots with up to 10 crossings, the braid word expressions have not been optimized yet. While no method has been proposed so far $[1,2]$ to find the most economic presentation of braids for knots, there appears a possibility to standardize systematically $[5,6]$ braid words for the simple knots.

\section{The torus-, the twist-, and the pretzel-knots}

We refer to the classical knot designation and to the link diagrams of Rolfsen [7]. We define the braid generator $b_{n}$ so that it produces a positive crossing $[2,8,9]$. As in [2], in a braid word, for the braid generator $b_{n}$, a factor $n$ will be written, and $n^{p}$ for $\left(b_{n}\right)^{p}$ with an integral power exponent $p$.

If the natural numbers $N$ and $p$ are relatively prime, the braid word

$$
w(N, p)=(123 \cdots(N-1))^{p}, \quad N, p,
$$

represents a torus knot $[2,3,10-13]$. The $(C, 2)$ torus knots $[7,10-14]$ have the number of crossings, or nodes, $C$, odd. If $C=2 n+1$, with natural $n$, the braid word for the $(C, 2)$ torus knot with positive crossings is $[2,3,11,12]$ :

$$
w\left(C_{1}\right)=w\left((2 n+1)_{1}\right)=1^{2 n+1}, \quad 2 n+1 .
$$


The next simple knots are the twist knots which have a plectonemic region with two string segments interwoven as in a braid of $C-2$ crossings, and are locked by an interlock with two crossings $[7,14,15]$.

A pretzel knot $[12,16-18]$ is composed of a row of $|b|+m$ parallel two-string braids. In the pretzel knot notation, $P\left(b ; q_{1}, \ldots, q_{m}\right)$, the integer $b$ separated by a semicolon gives the signed number of the one-crossing braids, the integers separated by a comma give the signed number of crossings in the braids $[12,17,18]$.

The braid closure words, $w\left(C_{i}\right)$, for twist knots, and several types of pretzel knots, will be written in a standardized form by the use $[2,3,5,8,9,19-21]$ of blocks $A(n)$ and $D(n)$, with natural $n>1$ :

$$
\begin{aligned}
& A(n)=23 \cdots(n-2)(n-1) n, \\
& D(n)=n(n-1)(n-2) \cdots 321 .
\end{aligned}
$$

The Conway notation [14], based on the concept of tangles and indicated in the Rolfsen [7] table of link diagrams, will follow, after a comma, the braid word for each knot, without specifying the sign of the crossings.

\section{Knots with an odd number of crossings}

In the classical knot labeling [7], when the crossing number $C$ is odd, the $(C, 2)$ torus knot is labeled $C_{1}$. The first type of twist knot, labeled $C_{2}$, has a plectonemic region interwoven as in the $(C-2,2)$ torus knot with $C-2$ crossings of the same sign, and is locked by an interlock with two crossings [7, 10-12, 15, 22-24]. A knot having an interlock with $b$ one-crossing braids $[7,12,14,17,18]$ is a pretzel knot $P(b ; C-|b|)$, as seen in Fig. 1 . The second type of twist knot which is locked by an interlock with three crossings [7] is a pretzel knot $P(3 ; C-3)$ and is labeled $C_{3}$. Knots with the crossing index $C=11$ are labeled here by the subscripts used by Conway [14] and by Perko [22]. Knots with larger crossing indices will be labeled by numbers of the Knotscape program [25].

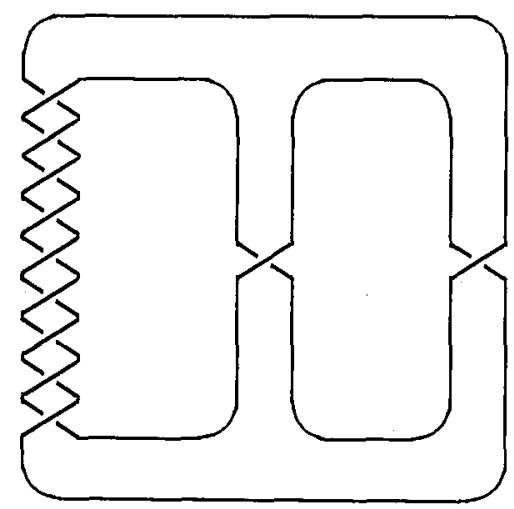

Fig. 1. The knot $11_{5}$ having an interlock with two crossings [12, 17, 18, 22], presented as a pretzel knot $P(2 ; 9)$. 
For the twist knots $C_{2}$ with an odd crossing number $C=2 n+1$, the braid words [2] can be written, by induction on the number of crossings, for natural $n>1$, as:

$$
w\left(C_{2}\right)=w\left((2 n+1)_{2}\right)=1 A(n) n D(n)(A(n))^{-1}, \quad(2 n-1) 2 .
$$

Since the length of each of the blocks $A(n)$ and $D(n)$ increases by one when $n$ increases by one, an increment of the crossing number $C$ by two increases the length of the braid word $[2,3]$ by three.

The braids for the second type of twist knots $C_{3}$ with the odd crossing number $C$ are 3-braids, i.e. have the braid index $N=3$. The braid words [2, 3], for natural $n>2$, are:

$$
w\left(C_{3}\right)=w\left((2 n+1)_{3}\right)=1^{2 n-2} 2^{2} 12^{-1}, \quad(2 n-2) 3 .
$$

The braid words for knots $7_{3}$ and $9_{3}$ conform in crossing signs to the respective knot diagrams of Rolfsen [7, 10]. Knots represented by 3 -braids $[1,5,6]$ have been illustrated also by diagrams of Akutsu et al. [3].

The braid words for the particular pretzel knots, see Fig. 1, can be inferred from the table of Jones [2] by induction. For a natural number $k$ we have:

$$
\begin{array}{ll}
w(P(2 k ; 5))=1 A(3) 32^{2 k} 1(A(3))^{-1}, & 2 k 5, \\
w(P(2 k ; 7))=1 A(4) 432^{2 k} 1(A(4))^{-1}, & 2 k 7, \\
w(P(2 k ; 9))=1 A(5) 5432^{2 k} 1(A(5))^{-1}, & 2 k 9, \\
w(P(2 k ; 11))=1 A(6) 65432^{2 k} 1(A(6))^{-1}, & 2 k 11 .
\end{array}
$$

For the pretzel knots with odd crossing numbers and with one one-crossing braid the braid words can be expressed:

$$
\begin{aligned}
& w(P(2 n-3,1,3)) \\
& =1 A(n-1)(n-1) n D(n)(A(n))^{-1}, \quad 2 n-313, \\
& w(P(2 n-5,1,5)) \\
& =1 A(n-2)(n-2)(n-1) n D(n)(A(n))^{-1}, \quad 2 n-515 \text {, } \\
& w(P(2 n-7,1,7)) \\
& =1 A(n-3)(n-3)(n-2)(n-1) n D(n)(A(n))^{-1}, \quad 2 n-717,
\end{aligned}
$$

for $n$ such that each braid generator number is $>1$.

At partition of the crossing number $C$ into more than three integers, it is convenient to use a natural number $k$, and to record

$$
\begin{array}{lr}
w(P(2 k, 3,3,3))=1^{4} 2^{3} 1^{-1} 32^{3} 3^{2 k-1}, & 2 k, 3,3,3, \\
w(P(2 k+1,2,3,3))=1^{4} 2^{2 k+1} 1^{-1} 32^{3} 3, & 2 k+1,2,3,3, \\
w(P(2 k+1,3,2,3))=1^{2 k+2} 2^{3} 1^{-1} 32^{3} 3, & 2 k+1,3,2,3, \\
w(P(2 k+1,4,3,3))=1^{4} 2^{2 k+1} 1^{-1} 3^{3} 2^{3} 3, & 2 k+1,4,3,3, \\
w(P(2 k+1,3,4,3))=1^{2 k+2} 2^{3} 1^{-1} 3^{3} 2^{3} 3, & 2 k+1,3,4,3 .
\end{array}
$$


Examples of braid closure words for the pretzel knots with three-crossing braids can be written:

$$
\begin{array}{ll}
w(P(3,3,3))=1^{2} 21^{-1} 32^{-1} 3243^{-1} 23^{2} 4^{-1} & \\
\quad=123^{2} 1^{-1} 23^{-1} 43^{-1} 213^{2} 4^{-1}, & 3,3,3, \\
w(P(5,3,3))=1234^{2} 34^{-1} 54^{-1} 3214^{2} 2^{-1} 3^{-1} 5^{-1}, & 5,3,3, \\
w(P(7,3,3))=12345^{2} 45^{-1} 65^{-1} 43215^{2} 2^{-1} 3^{-1} 4^{-1} 6^{-1}, & 7,3,3, \\
w(P(9,3,3))=123456^{2} 56^{-1} 76^{-1} 543216^{2} 2^{-1} 3^{-1} 4^{-1} 5^{-1} 7^{-1}, & 9,3,3, \\
w(P(7,3,5))=1^{2} 21^{-1} 32^{-1} 45^{-1} 46^{2} 54^{-1} 6^{-1} 76^{-1} 54326^{2} 54^{-1} 7^{-1}, & 7,3,5, \\
\quad=12345^{-1} 46^{2} 54^{-1} 6^{-1} 76^{-1} 543216^{2} 52^{-1} 3^{-1} 4^{-1} 7^{-1}, & \\
w(P(3,3,3,3,3)) &
\end{array}
$$

The pretzel knot $P(7,3,5)$, presented in Fig. 2 , is a non-invertible knot with the lowest possible values of the indices $[16,26]$.

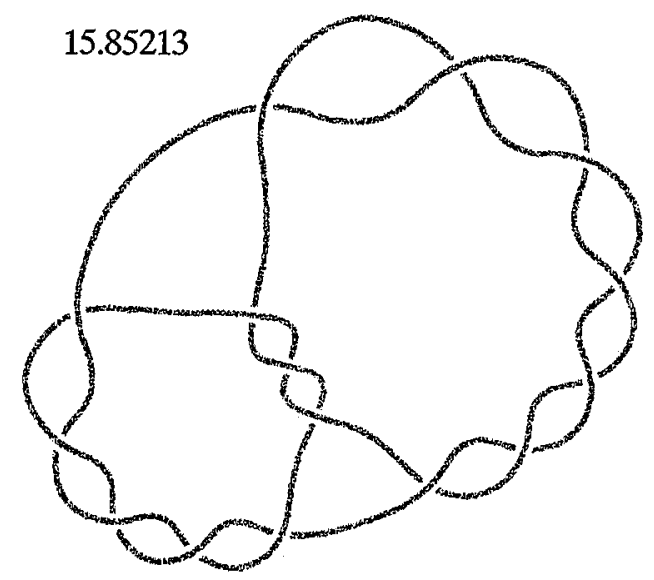

Fig. 2. The non-invertible $[16,26]$ knot $P(7,3,5)$.

Braid words for the pretzel knots with the five-crossing braids can be written:

$$
\begin{aligned}
& w(P(5,5,3)) \\
& \quad=1231^{-1} 4^{-1} 35^{2} 43^{-1} 5^{-1} 65^{-1} 43215^{2} 43^{-1} 6^{-1}, \\
& w(P(5,5,5)) \\
& \quad=1234^{-1} 35^{-1} 4^{-1} 6^{2} 54^{2} 3214^{-1} 6^{-1} 76^{-1} 56^{2} 542^{-1} 3^{-1} 7^{-1},
\end{aligned}
$$




\section{Knots with an even number of crossings}

If the crossing number $C$ of knot is even, the first type of twist knot which has a plectonemic region interwoven as in a torus knot with $C-2$ crossings, and is locked by an interlock with two crossings of the other sign [7, 15, 23,24], is a pretzel knot $P(2 ; C-2)$, and is labeled $C_{1}$. The second type of twist knot, locked by an interlock with three crossings [7], is a $P(C-3,1,2) \mathrm{knot}$, and is labeled $C_{2}$. The third type of twist knot, labeled $C_{3}$, locked by an interlock with four crossings [7], is a $P(4 ; C-4)$ knot, see Fig. 3 .

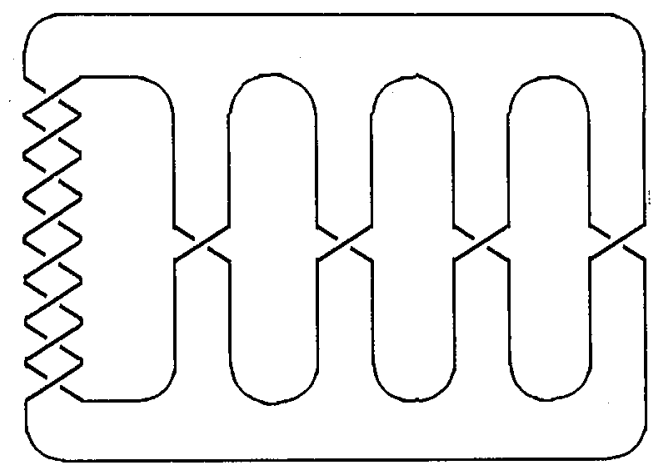

Fig. 3. The knot $P(4 ; 8)$ has Knotscape No. 12.1166 .

The braid words [2] for the twist knots $C_{1}$, with an even crossing number $C=2 n$, can be written, by induction on the number of crossings, for natural $n>2$, as:

$$
\begin{aligned}
w\left(C_{1}\right) & =w\left((2 n)_{1}\right) \\
= & 1 A(n-1) n^{-1} D(n-1)(A(n-1))^{-1} n^{-1}, \quad(2 n-2) 2 .
\end{aligned}
$$

An increment of the crossing number $C$ by two increases the length of the braid word $[2,3]$ by three.

The braids for the second type of twist knots $C_{2}$, with the even crossing number $C$, are 3 -braids. The braid words [2,3], for natural $n>2$, are:

$$
w\left(C_{2}\right)=w\left((2 n)_{2}\right)=1^{2 n-3} 2^{-1} 12^{-1}, \quad(2 n-3) 12
$$

The braid words [2] for the third type of twist knots $C_{3}$, with the even crossing number $C$, can be written, by induction on the number of crossings, for natural $n>3$, as:

$$
\begin{aligned}
& w\left(C_{3}\right)=w\left((2 n)_{3}\right) \\
& =1 A(n-1) n^{-1}(n-1)^{-1} D(n-2)(A(n-1))^{-1} n^{-1}, \quad(2 n-4) 4 .
\end{aligned}
$$

An increment of the crossing number $C$ by two increases the length of the braid word $[2,3]$ by three.

For the pretzel knots with even crossing numbers and with one one-crossing braid the braid words can be expressed: 


$$
\begin{aligned}
& w(P(2 n-4,1,3)) \\
& \quad=1 A(n-2)(n-1)^{-1} D(n-2)(n-1)^{-2}(A(n-1))^{-1}, \quad 2 n-413, \\
& w(P(2 n-6,1,5)) \\
& \quad=1 A(n-3)(n-2)^{-1} D(n-3)(n-2)^{-4}(A(n-2))^{-1}, \quad 2 n-615, \\
& w(P(2 n-8,1,7)) \\
& \quad=1 A(n-4)(n-3)^{-1} D(n-4)(n-3)^{-6}(A(n-3))^{-1}, \quad 2 n-817,
\end{aligned}
$$

for $n$ such that each braid generator number is $>1$.

At partition of the crossing number $C$ into three, and more integers, it is convenient to use natural numbers $k, l$, and to record the words:

$$
\begin{aligned}
& w(P(2 k+1,2 l+1,2)) \\
& =1^{2 k+1} 2^{-1} 1^{2 l+1} 2^{-1}, \\
& 2 k+1,2 l+1,2 \\
& w(P(2 k+1,2 l+1,4)) \\
& =1^{2 k+1} 2^{-1} 1^{2 l+1} 32^{-1} 3^{-2}, \quad 2 k+1,2 l+1,4, \\
& w(P(2 k+1,2 l+1,6)) \\
& =1^{2 k+1} 2^{-1} 1^{2 l+1} 432^{-1} 3^{-1} 4^{-2} 3^{-1}, \quad 2 k+1,2 l+1,6, \\
& w(P(2 k+1,2 l+1,8)) \\
& =1^{2 k+1} 2^{-1} 1^{2 l+1} 5432^{-1} 3^{-1} 4^{-1} 5^{-2} 4^{-1} 3^{-1}, \quad 2 k+1,2 l+1,8, \\
& w(P(2 k+1,2 l+1,10)) \\
& =1^{2 k+1} 2^{-1} 1^{2 l+1} 65432^{-1} 3^{-1} 4^{-1} 5^{-1} 6^{-2} 5^{-1} 4^{-1} 3^{-1}, \quad 2 k+1,2 l+1,10 .
\end{aligned}
$$

The diagram of the pretzel knot $P(5,5,6)$ in Fig. 4 has been drawn, like the knot in Fig. 2, by the Knotscape program [25] which allows one to draw knots with up to 16 crossings.

At partition of $C$ into five integers we record:

$$
\begin{aligned}
& w(P(2 k+1,3,2,3,3)) \\
& =123^{3} 2^{2} 1^{-1} 2^{2 k+1} 4^{-1} 3^{-1} 2^{3} 34^{-1}, \quad 2 k+1,3,2,3,3, \\
& w(P(2 k+1,2,3,3,3)) \\
& =123^{3} 2^{2} 1^{-1} 2^{3} 4^{-1} 3^{-1} 2^{2 k+1} 34^{-1}, \quad 2 k+1,2,3,3,3, \\
& w(P(2 k+1,3,4,3,3)) \\
& =123^{3} 2^{2} 1^{-1} 2^{2 k+1} 4^{-1} 3^{-1} 2^{3} 354^{-1} 5^{-2}, \quad 2 k+1,3,4,3,3, \\
& w(P(2 k+1,4,3,3,3)) \\
& =123^{3} 2^{2} 1^{-1} 2^{3} 4^{-1} 3^{-1} 2^{2 k+1} 354^{-1} 5^{-2}, \quad 2 k+1,4,3,3,3 .
\end{aligned}
$$




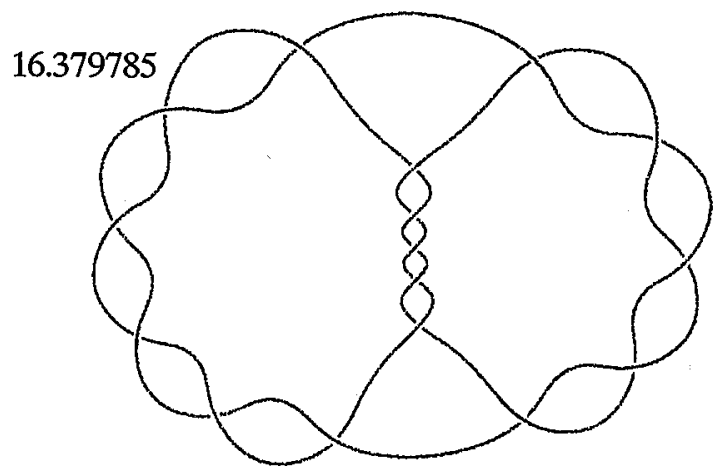

Fig. 4. The knot $P(5,5,6)$.

The braid words recorded for the mirror image of the knot diagrams of Rolfsen [7] will conform in the crossing sign to Rolfsen diagrams if the sign of the power exponent at each braid generator would be reversed, or the braid generator $b_{n}$ would be defined $[1,3]$ so that it yields a negative crossing.

\section{Concluding remarks}

The braid words can be transformed according to the rules known for the closed braid moves [1, 2, 8, 10-13].

The expression of braid words for the pretzel knots in a standardized form $[5,6,9]$ enables one to see the regular pattern of the words, and thus to write the braid words of these knots for general values of the crossing number [27-29], and to locate a desired pretzel knot in such tabulation e.g. as provided by the Knotscape program [25].

In the laboratory synthesis of long organic molecular chains, in particular of polyether ladders, a synthesis of the knot $4_{1}$ was discussed [30]. The knotted carbynes, i.e. carbon allotropes with pure $s p$-hybridization, were considered, up to the $6_{3}$ knot, in the $a b$ initio calculations [31], to estimate their stability and the
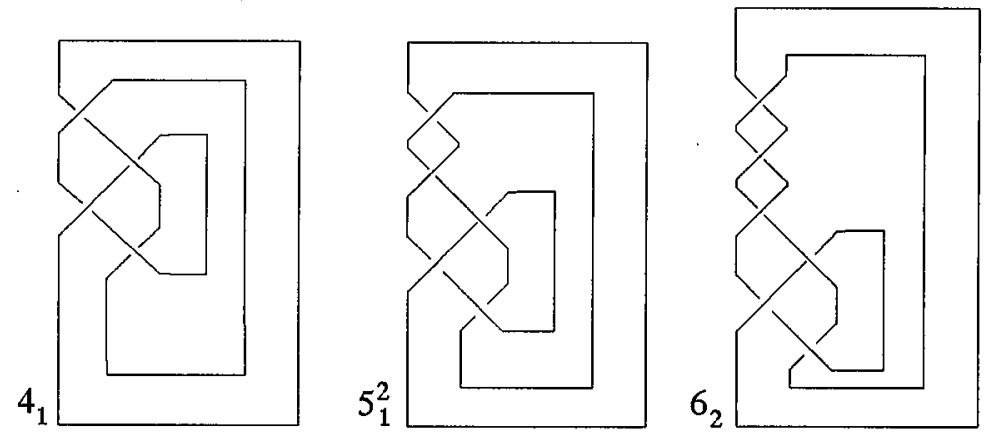

Fig. 5. Braid closures relevant to successive rounds of tangle addition in the processive recombination of DNA. 
number of their NMR lines. In the processive recombination of a closed DNA ring, products are created in a sequence with an increasing number of supercoil crossings [32-37]. The recombination of the DNA ring by a resolvase enzyme yields in successive rounds of tangle addition: the two-component link $2_{1}^{2}$, the twist knot 41 , the two-component link $5_{1}^{2}$, and the twist knot $6_{2}$, shown by diagrams in the review [34] and in Fig. 5. The review [34] reports also the results of recombination by the Gin system of bacteriophage Mu on a knotted DNA substrate. From the knotted DNA substrates, as products are found, inter alia, the knots: $3_{1}, 4_{1}, 5_{2}, 6_{1}, 7_{2}$, and $7_{5}$. Based on an abundant supply of illustrative examples, the review [34] asserts: "Biologically, the two most important families of knots and catenanes are the torus and twist families". A standardized form of braid words is useful for presentation of these links in a discussion of their recombination processes.

Private communications of Professors J. Hoste, M.B. Thistlethwaite, J.R. Weeks, and J. Konarski are acknowledged.

\section{References}

[1] J.S. Birman, Bull. Am. Math. Soc. 28, 253 (1993).

[2] V.F.R. Jones, Ann. Math. 126, 335 (1987).

[3] Y. Akutsu, T. Deguchi, M. Wadati, J. Phys. Soc. Jpn. 56, 3464 (1987).

[4] P. Vogel, Comment. Math. Helvet. 65, 104 (1990).

[5] T. Randrup, P. Røgen, Arch. Math. 68, 252 (1997).

[6] S. Kamada, Michigan Math. J. 45, 189 (1998).

[7] D. Rolfsen, Knots and Links, Publish or Perish, Inc., Berkeley (California) 1976.

[8] H.R. Morton, H.B. Short, J. Algorithms 11(2), 117 (1990).

[9] E.A. Elrifai, H.R. Morton, Quart. J. Math. Oxford (2),45, 479 (1994).

[10] G. Burde, H. Zieschang, Knots, W. de Gruyter, New York 1985.

[11] C.C. Adams, The Knot Book, W.H. Freeman and Co., New York 1994.

[12] A. Kawauchi, A Survey of Knot Theory, Birkhäuser, Basel 1996.

[13] K. Murasugi, Knot Theory and its Applications, Birkhäuser, Basel 1996.

[14] J.H. Conway, in: Computational Problems in Abstract Algebra, Proc. Conf. Oxford 1967, Ed. J. Leech, Pergamon Press, Oxford 1970, p. 329.

[15] E.P. Klassen, Trans. Am. Math. Soc. 326, 795 (1991).

[16] H.F. Trotter, Topology 2, 275 (1964).

[17] A. Kawauchi, Kobe J. Math. 2, 11 (1985).

[18] D.S. Silver, S.G. Williams, Trans. Am. Math. Soc. 351, 3243 (1999).

[19] E. Artin, Ann. Math. 48, 101, 643 (1947).

[20] H. Nencka, Contemp. Math. 283, 221 (1999).

[21] P.R. Cromwell, Proc. Lond. Math. Soc. (3),67, 334 (1993).

[22] K.A. Perko Jr., Topology Proc. 7(1), 109 (1982).

[23] W.B.R. Lickorish, K.C. Millet, Topology 26, 107 (1987).

[24] P.J. Callahan, J.C. Dean, J.R. Weeks, J. Knot, Theory Ramif. 8, (3)279 (1999). 
[25] J. Hoste, M. Thistlethwaite, J.R. Weeks, The Mathematical Intelligencer 20, (4)33 (1998).

[26] M. Kouno, K. Motegi, Math. Proc. Camb. Philos. Soc. 115, 219 (1993).

[27] M. Suffczyński, Acta Phys. Pol. A 93, 679 (1998).

[28] S. Boyer, T. Mattman, X. Zhang, in: Knots '96, Ed. S. Suzuki, World Scientific, River Edge (NJ) 1997, p. 159.

[29] R.A. Landvoy, Topology Appl. 83(2), 135 (1998).

[30] D.M. Walba, T.C. Homan, R.M. Richards, R.C. Haltiwanger, New J. Chem. 17, 661 (1993).

[31] J.C. Dobrowolski, A.P. Mazurek, Int. J. Quantum Chem. 70, 1009 (1998).

[32] P. Dröge, N.R. Cozzarelli, Methods in Enzymology 212, 120 (1992).

[33] C. Ernst, D.W. Sumners, Math. Proc. Camb. Philos. Soc. 108, 489 (1990).

[34] D.W. Sumners, C. Ernst, S.J. Spengler, N.R. Cozzarelli, Quart. Rev. Biophys. 28, (3) 253 (1995).

[35] G. Buck, J. Simon, in: Lectures at Knots 96, Ed. S. Suzuki, World Scientific, Singapore 1997, p. 219.

[36] M. Suffczyński, Polish J. Chem. 69, 157 (1995).

[37] M. Suffczyński, in: Symmetry and Structural Properties of Condensed Matter, Proc. Conf. Zajaczkowo 1996, Eds. T. Lulek, W. Florek, B. Lulek, World Scientific, Singapore 1997, p. 435. 